\title{
Direct measurement of torsional properties of single
}

\section{fibers}

\author{
Dabiao Liu ${ }^{1,2,3}$, Kai Peng ${ }^{1,2}$, Yuming $\mathrm{He}^{1,2, *}$ \\ ${ }^{1}$ Department of Mechanics, Huazhong University of Science and Technology, Wuhan 430074, China \\ ${ }^{2}$ Hubei Key Laboratory of Engineering Structural Analysis and Safety Assessment, Wuhan 430074, China \\ ${ }^{3}$ School of Physics and Astronomy, Queen Mary University of London, London E1 4NS, UK
}

*Corresponding author.

Prof. Yuming He

Department of Mechanics

Huazhong University of Science and Technology, Wuhan 430074, China

TEL: +86 $2787543438 ;$ FAX: +86 2787544882

E-mail: ymhe@hust.edu.cn

\begin{abstract}
In order to characterize the torsional behavior of micron-scale specimens, a direct technique is established based on the principle of torsion balance. The technique applies twist to the specimen and balances the resulting torque against a torsion wire of known torsional rigidity. The torsional rigidity of the torsion wire is determined by a torsion pendulum. To measure the rotation of the torsion wire, a sensitive angle detector, comprised of a thin cross-beam attached between the torsion wire and the fiber specimen and a laser displacement sensor, is developed. The presented technique permits the measurement of torque in single fibers as low as $10^{-9} \mathrm{~N} \bullet \mathrm{m}$ with a reasonable resolution. Using this technique, torsion tests on micro-diameter copper wires, silver wires and carbon fibers were performed. The longitudinal shear modulus and other torsional properties of these samples, such as yielding shear stress, were obtained.
\end{abstract}

Keywords: Torsion balance; Torsion pendulum; Micro-diameter; Single fiber; Shear modulus 


\section{Introduction}

Quasi-static torsion testing on small specimens has been recognized as one of excellent approaches to study the mechanical behaviors of small-scale specimens, from elastic deformation, through yielding, to the strain-hardening regime, whereby the strain gradients are naturally generated in plasticity [1-3]. Generally speaking, the development of torsion testing techniques of single fibers/wires is motivated by at least two reasons: the recent interests in the size-dependent plasticity at small scales [4], and the requirement for the shear properties of single high-performance fibers in textile [5] and in the design of high strength composites [6]. However, compared to the tensile techniques at small scales that have been mature commercially $[7,8]$, the development of torsion techniques at small scales is rather slow due to several challenges [1], including the difficulty in detecting micro-torque and in handling the small specimens. Over the past decades, a series of non-standard techniques for measuring the torsional properties of single fibers/wires have been proposed. Most of them are based upon the principle of torsion balance [9] that can be traced back to the landmark works by Charles Coulomb [10] and Henry Cavendish [11]. In fact, the torsion-balance devices are ubiquitous in experimental physics, measurement science and various other fields of engineering [9]. Here, we only focus on the torsion-balance techniques applied in the torsional characterization of single fibers, wires and yarns.

In an ideal torsion balance for thin fibers, the specimen is generally fixed between a twisting head at its lower end and a torsion wire of known properties at its upper end. The upper end of the torsion wire can be fixed or be rotated manually. A pointer, or other indicating device, is positioned between the specimen and the torsion wire. As the specimen is twisted, the torque applied on the specimen can be determined by measuring the rotation of the indicating device. Yet, this technique has not been widely used due to the difficult in its implementation. In 1923, Peirce[12] developed a magnetic torsion tester for the measurement of torsional relaxation of yarns. In this apparatus, the author employed a known magnetic field to measure the torque in the specimen that was suspended from a torsion head. Following the similar principle, Huan et al. [13] recently adopted a coil placed in the radial magnetic field to measure the torque of micro-scale specimens. Additionally, an 
optical lever system has been widely used to measure the rotation of the indicating device $[5$, 6, 14-16]. For example, Mitchell and Feughelman [14] used a photographic film to record the movement of the light image in the optical-lever system. Postle et al. [17] adopted a lamp-and-scale arrangement to measure the torque in a twisted yarn. Skelton [18] used a circular scale to detect the angular rotation of the mirror that was attached between the torsion wire and the specimen. More recently, Behlow et al. [6] proposed a design for the measurement of torque using a sensitive PSD-based optical detector that tracks a torsion reference. Rather than using an optical lever system, Kawabata [19] adopted a pair of linear differential transformers to detect the rotation of the torsion wire. Mccord et al. [20] employed an optical encoder module and a slot incremental code wheel disk to measure the rotation of the torsion wire. The main issue of these two methods is that the moment of inertia attached to the torsion wire seems too large to give an instantaneous measurement of torque. Another problem is the influence of the friction on the torque measurement introduced by jewel bearing $[6,13]$ or air bearing $[20]$.

On the other hand, in 1994, Fleck et al. [3] developed a screw-driven torsion balance to perform torsion tests on polycrystalline copper wires ranging in diameters from 12 to $170 \mu \mathrm{m}$ for studying the strain-gradient effect in plasticity. Since Fleck et al.'s pioneering work [3], torsion of thin metallic wires has been recognized as a benchmark experiment in micromechanics [4]. However, it is hard to distinguish the elastic range from the normalized toque-twist curves in Fleck et al.'s experimental data due to the poor resolution of their apparatus. This drawback has motivated many other researchers to develop a range of new techniques for wire torsion, including the improved fiber-based torsion balance $[1,21,22]$, AFM-based torsion balance [21, 23], and load-unload technique $[2,24,25]$. However, most of techniques aforementioned above are unable to test single fibers/wires with diameter less than ten microns $[21,23]$. And the calibration methods are different from one to another, making the comparability and traceability of the experimental results worse.

As reviewed by Liu et al. [26], another well-known method for measuring the torsional properties of single fibers is the torsion-pendulum technique. Although the torsion-pendulum approach is simple in design and rapid in application, the information it provided is severely limited since the rigidity is measured for only small strains. It is generally 
unable to provide the information related to plasticity, creep or hysteresis [27]. The torsion-balance technique introduced here overcomes many of these limitations, and can be used to measure the torsional yield and plasticity information as well as shear stress-strain response.

In this paper, a new torsion tester with high resolution (up to $10^{-10} \mathrm{~N} \cdot \mathrm{m}$ ) and reasonable precision (about $8 \%$ ) for measuring the torsional properties of individual fibers is established based on the principle of torsion balance. In this tester, a fiber specimen is subjected to a given twist and the resulting torque is measured by a torsion (tungsten) wire of known torsional rigidity. The inserted twist can be controlled accurately so that both the shear modulus and the shear yield strength of specimens can be determined. The sensitivity and reproducibility of the technique is discussed. Finally, the proposed technique is applied to investigate the torsional properties of micro-diameter copper and silver wires, and carbon fibers.

\section{Experimental details}

\subsection{Torsion balance setup}

Based on the static torsion-balance principle, a torsion tester has been established for measuring the toque-twist curves of an individual fiber, as shown in Fig. 1(a). It is designed to balance the torque acting on the specimen against the torque of a torsion wire (i.e., a torque meter). In this arrangement, a fiber specimen is fixed between a twisting head at its lower end and a torsion wire (a tungsten wire used here) of known torsional rigidity at its upper end. The upper end of the torsion wire of length $l$ and of diameter $d$ is glued, using cyanoacrylate glue, onto a thin rigid beam mounted on a three-dimensional translation stage. The lower end of the torsion wire is attached to a stiff and light cross-beam (about $0.4 \mathrm{~g}$, acted as an indicating device) which is connected to the upper end of the fiber specimen. The lower end of the fiber specimen is attached to a tensioning mass made of one or two washers for giving a constant stress of about 2-3 MPa, far below its yield strength, during the measurement. The tension mass is inserted into a slot (i.e. twisting head) to prevent lateral 
movement while still allowing the mass to slide freely in the vertical direction, as shown in Fig. 1(a). The twisting head is mounted on the stepper motor (i.e. turntable) located at a three-dimensional translation stage. A constant rate of twist is applied to the specimen by the stepper motor.

As the specimen is twisted, the applied torque in the fiber specimen is transmitted to the torsion wire through the stiff cross-beam. Twist of the specimen leads to a rotation of the cross-beam resulting in a torque in the torsion wire. At equilibrium, the torque in the torsion wire is equal and opposite to that in the specimen, i.e.,

$$
T=M=K \theta
$$

where $\theta$ is the rotation of the cross-beam, and $K$ is the torsion constant (torque per unit twist) of the torsion wire. The angular displacement $\theta$ is detected by an optical detection system (see Fig. 1(b) for details), which is calculated as

$$
\theta=\arctan (\delta / L)
$$

where $\delta$ is the distance of the laser spot deviated from the initial position, and $L$ is the horizontal distance between the laser spot and the torsion wire centered in the cross-beam. The twist of the specimen is the difference between the torsion angle applied by the stepper motor and $\theta$. Then the relative twist of the two ends of the specimen is

$$
\varphi=\psi-\theta
$$

where $\psi$ is the twist angle given by the stepper motor. If a suitable torsion wire is used, the value of $\theta / \psi$ can be less than $10^{-4}$. Therefore, one can adopt the twist angle provided by the stepper motor, $\psi$, to denote the twist inserted into the specimen, i.e. $\varphi \approx \psi$.

If the value of $K$ in Eq. (1) is known, the torque $T$ can be obtained. It is known that the value of $K$ depends on the length, $l$, and the torsional rigidity, $G I_{p}$, of the torsion wire, i.e.

$$
K=\frac{G I_{p}}{l}=\frac{G \pi d^{4}}{32 l}
$$

where $G$ is the shear modulus, $d$ is the diameter of the torsion wire, and $I_{p}$ is its moment of inertia. The determination of $G$ can be achieved by means of a torsion pendulum (see Section 2.3 for details). Finally, according to Eqs.(1)-(3), one can obtain the torque-twist data continuously for the specimen. The sensitivity of the apparatus can be 
altered by exchanging the torsion wire, for one of greater or less torsional rigidity. Generally, the torque per unit angular displacement of the torsion wire should be approximately ten times the magnitude of the greatest value of toque applied in the fiber specimen [5].
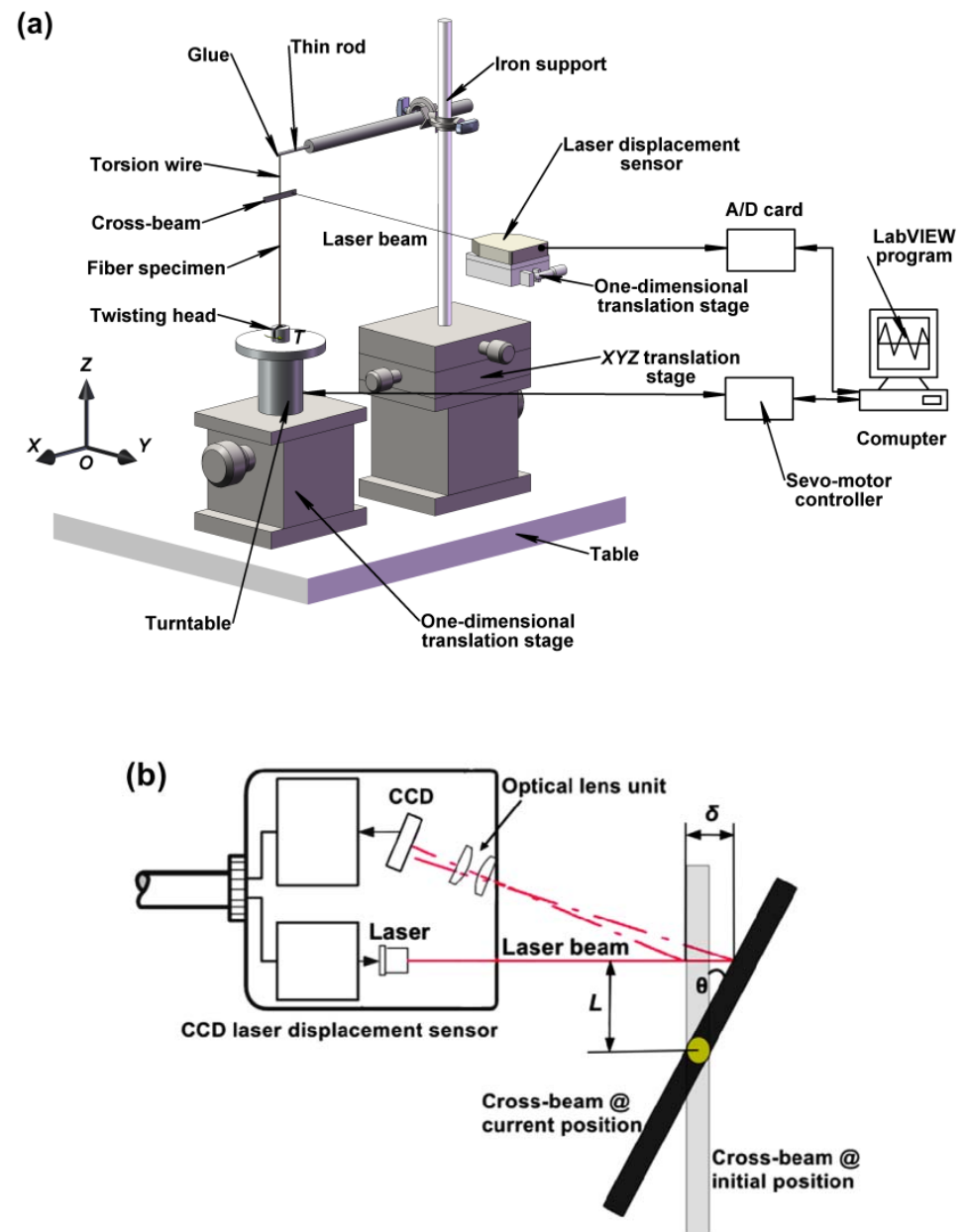

FIG. 1. (a) Schematic diagram of the torsion tester; (b) Diagram of the optical detection system for detecting the rotation angle of the torsion wire.

The design is realized within the previous self-developed torsion testing instrument $[1,22]$. The rotation rate provided by the stepper motor is ranging from $0.05236 \mathrm{rad} / \mathrm{s}$ to 0.5236 $\mathrm{rad} / \mathrm{s}$. The minimum angular increment of stepper of is $0.0314 \mathrm{rad}$, but if necessary, a servomotor could be substituted to provide an increased resolution or rotation rate. The optical detector for measuring the angular displacement of the torsion wire consists of a laser displacement sensor (Keyence, LK-G80) with a resolution of $0.1 \mu \mathrm{m}$ and the cross-beam attached between the torsion wire and the specimen. The cross-beam is initially adjusted to be perpendicular to the laser beam emitted from the laser displacement sensor. The laser 
beam is reflected off the cross-beam and collected by a CCD within the displacement sensor, as illustrated in Fig. 1(b). The experiment control and data acquisition for the torsion testing is automated through a LabVIEW (National Instruments Corp.) program in a computer, which provides for control of a range of experimental parameters, such as the rotating speed, twist amplitude, cyclic numbers (if cyclic torsion is desired). During testing, the data are displayed real-timely on a strip recorder onscreen and are called to a torque-twist graph upon completion.

\subsection{Calibration of the torsion wire}

The calibration of the torsion wire is crucial for the torsion testing. Several researchers determined the torsional rigidity of the torsion wire by applying a known torque to the wire $[3,28]$. This was achieved by using a dead weight suspended over a pulley arrangement $[3$, 28]. Such a calibration method has been proved to be unsatisfactory because of the friction in the pulley used to redirect the load. Here, we adopt an improved torsion pendulum based on image processing [27] to determine the shear modulus of the torsion wire accurately, as shown in Fig.2(a). Here, we chose tungsten wires ranging in diameter from 15 to $40 \mu \mathrm{m}$ as the torsion wires. The real diameter of each tungsten wire was determined with a scanning electron microscopy (SEM), see Fig. 4(a) for example. A length of tungsten wire was suspended at one end, and two washers with a suitable mass were bonded to the other end. The oscillation motion of the suspended mass was recorded with a video camera overhead. An image processing method has been developed to calculate the twist angle of the pendulum. The oscillation frequency was then determined by the Fast Fourier Transform (FFT). The shear modulus of the tungsten wire can be calculated as $[26,27]$

$$
G_{\text {torsin wire }}=\frac{\pi m l^{*} f^{2}\left[8\left(D_{0}^{2}+D_{i}^{2}\right)+\frac{32}{3} h^{2}\right]}{d^{4}}
$$

Here, $m$ is the mass, $h$ is the thickness, $D_{o}$ and $D_{i}$ are the outer and inner diameters of the washers, respectively; $l^{*}$ is the wire length, $d$ is the wire diameter, and $f$ is the torsion oscillation frequency of the pendulum. 
We take the torsion pendulum testing on the tungsten wire of diameter $30.53 \pm 0.10 \mu \mathrm{m}$ as an example. In this test, two steel washers clamped and bonded with a small amount of glue at the free end were used as the rotating mass. The washers were with the following parameters: $D_{o}=12.34 \mathrm{~mm}, D_{i}=6.36 \mathrm{~mm}, H=2.10 \mathrm{~mm}$, and $m=1.1885 \mathrm{~g}$. The detailed description of the torsion pendulum is given by $\mathrm{Yu}$ et al. [27] As shown in Fig. 2(a), by providing and releasing a small initial torque, we set the pendulum under a free torsional oscillation. A typical oscillation curve is given in Fig. 2(b). The corresponding fundamental oscillation frequency is determined by FFT, which is found to be $0.520 \mathrm{~Hz}$, as shown in Fig. 2(c). The length of the tungsten wire is $64.52 \mathrm{~mm}$. Then, the shear modulus of the tungsten wire is calculated as $G_{\text {torsion wire }}=121.43 \pm 3.0 \mathrm{GPa}$, which is in agreement with the results in literatures [26, 27]. Substituting the values of $G$ and other parameters into Eq. (4), one can obtain the torsional constant of the torsion wire.

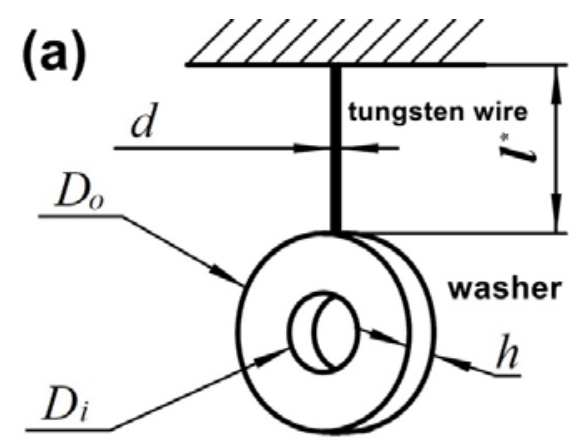

(b)

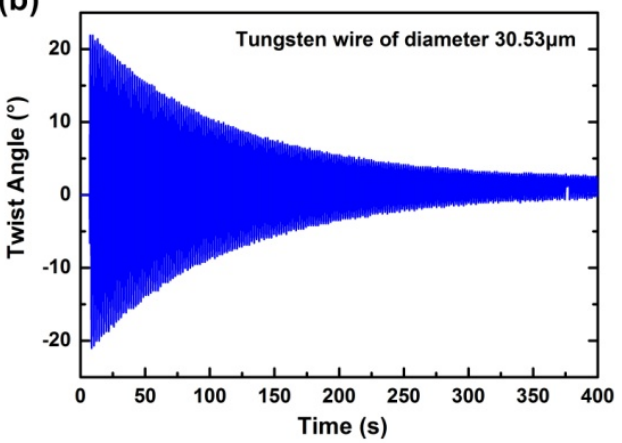

(c) $10^{2}$

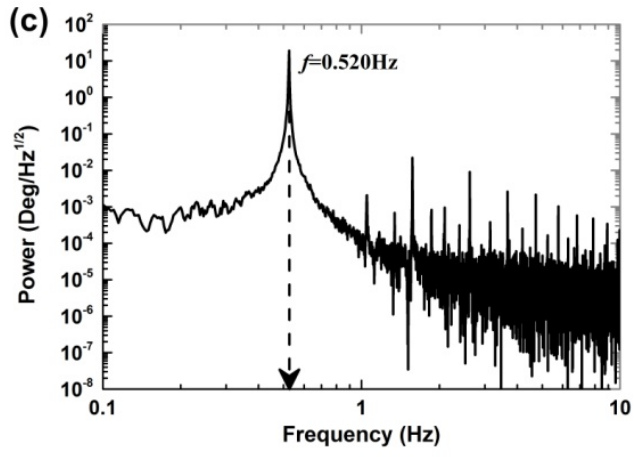

FIG. 2. Calibration of the torsion wire. (a) Diagram of a torsional pendulum; (b)Typical torsional oscillation data of the Tungsten wire; (c) The power spectrum generated by FFT with a sampling interval of $0.04 \mathrm{~s}$. 


\subsection{Preparation of the specimens}

The fiber specimens were prepared by following procedures. A single strand of the fiber was isolated with the aid of an optical microscope (Keyence VHX-500FE). In order to avoid damage, the two ends of the fiber specimen of length about $15 \mathrm{~cm}$ were glued to two V-holders on a paperboard, as shown in Fig.3 (a). Similarly, a torsion wire of length about $5 \mathrm{~cm}$ was also bonded on another paperboard in the same way (Fig.3 (b)). These two paperboards were then placed together in a way that the specimen and the torsion wire were aligned to each other, as illustrated in Fig. 3(c). Afterwards, the cross-beam, consisting of an aluminum foil with a thickness of $200 \mu \mathrm{m}$, a height of $8 \mathrm{~mm}$ and a length of $30 \mathrm{~mm}$, was bonded to a suitable position of the torsion wire and the specimen for fixing them together. With the cross-beam being a dividing line, we cut a segment of specimen at the left and a segment of torsion wire at the right (see Fig. 3(d)), respectively. Finally, a washer with a suitable mass was attached to the end of the specimen, and the torsion wire was glued to a thin rigid beam, as shown in Fig. 3(e).

(a)

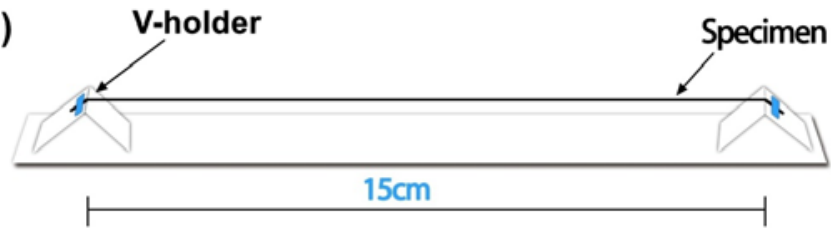

(b)

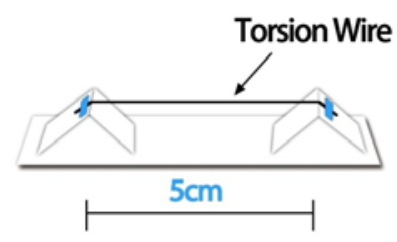

(c)

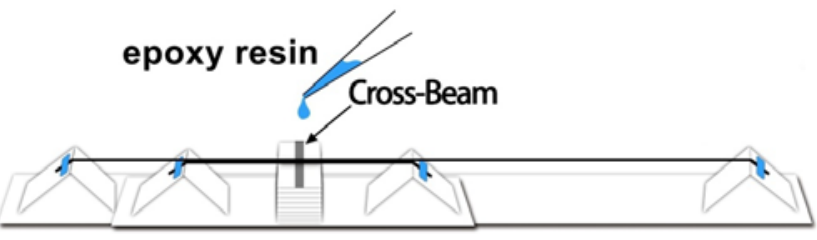

(d)

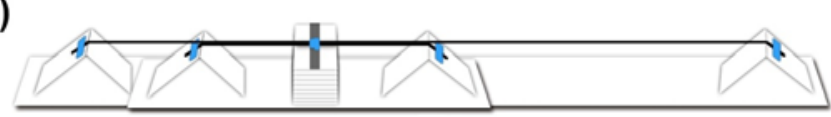

(e) Torsion wire

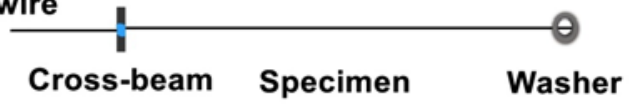

FIG. 3. Schematic illustration for preparation of the fiber specimens. 
Here, three types of fibers/wires were chosen for testing, i.e. copper wire (99.999\% purity, Henan Youk Electronic Material Co., Ltd., China), silver wire (>99.99\% purity, MK Electron(Kunshan) Co., Ltd.), and carbon fiber (T300, PAN, Toray Industries, Inc.). The diameters of the specimens and the torsion wires were measured by a SEM (Quanta 3D Dual Beam system FIB-SEM), as shown in Fig. 4.
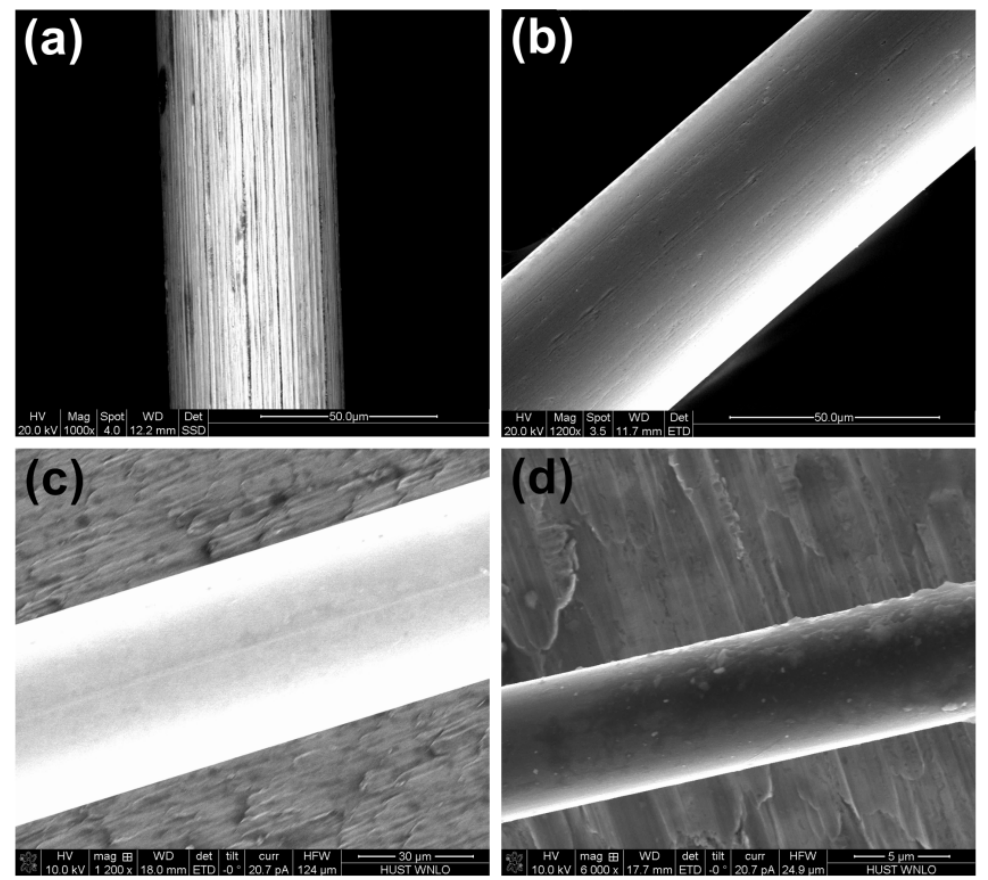

FIG. 4. SEM images of torsion wire and fiber specimens: (a) Tungsten wire of diameter $30.53 \pm 0.10 \mu \mathrm{m}$, (b) copper wire of diameter $49.89 \pm 0.27 \mu \mathrm{m}$, (c) silver wire of diameter $49.57 \pm 0.25 \mu \mathrm{m}$, and (d) T300 carbon fiber of diameter $7.04 \pm 0.02 \mu \mathrm{m}$. Silver wires and carbon fibers used here are the same as those in Reference [27].

\section{Evaluation of the technique}

To test the sensitivity and reproducibility of the torsion tester, three repeated measurements were performed on $18 \mu \mathrm{m}$-diameter polycrystalline copper wires in elastic range, as shown in Fig. 5. The torsion data are displayed in the form of surface shear stress $\tau=\frac{2 T}{\pi a^{3}}$ versus surface shear strain $\gamma=\kappa a$. Here, $T$ is the torque, $a$ the wire radius, and $\kappa$ the twist per unit length. The experimental curves exhibit a reasonable repeatability. We used the mean curve as a representative response (see Fig. 5), in which the error bar 
marked on the mean curve is $\pm 2 \mathrm{MPa}$. The corresponding shear modulus is calculated as $54.11 \pm 2.14 \mathrm{GPa}$, as listed in Table 1 . The fluctuations in the curves are mainly due to the misalignment between the specimen and the twisting head, as shown in Fig. 6. As pointed out by Walter and Kraft [23], it is hard to align the specimen perfectly into the twisting head. Therefore, the cross-beam moves around a circular track during one rotation (see Fig. 6(a)). Such a motion leads to an alternating signal in the angular displacement measurement of the torsion wire, as well as in the torque measurement, as illustrated in Figs. 6 (b) and (c). One can see that the closer is the cross-beam to the fixed end of the torsion wire, the less is the influence of misalignment on the torque measurement. Therefore, we adopted the torsion wire of length in the range $10-25 \mathrm{~mm}$ throughout testing, which was much shorter than the gauge length of the specimens. In addition, the roughness of the cross-beam also has an influence on the torque signal, which turns out to be much smaller than that caused by the misalignment. According to Eqs. (1) and (4), one can see that the measured torque is strongly dependent on the wire diameter. That is, there is a dramatically decrease in torque with decreasing the fiber/wire diameter.

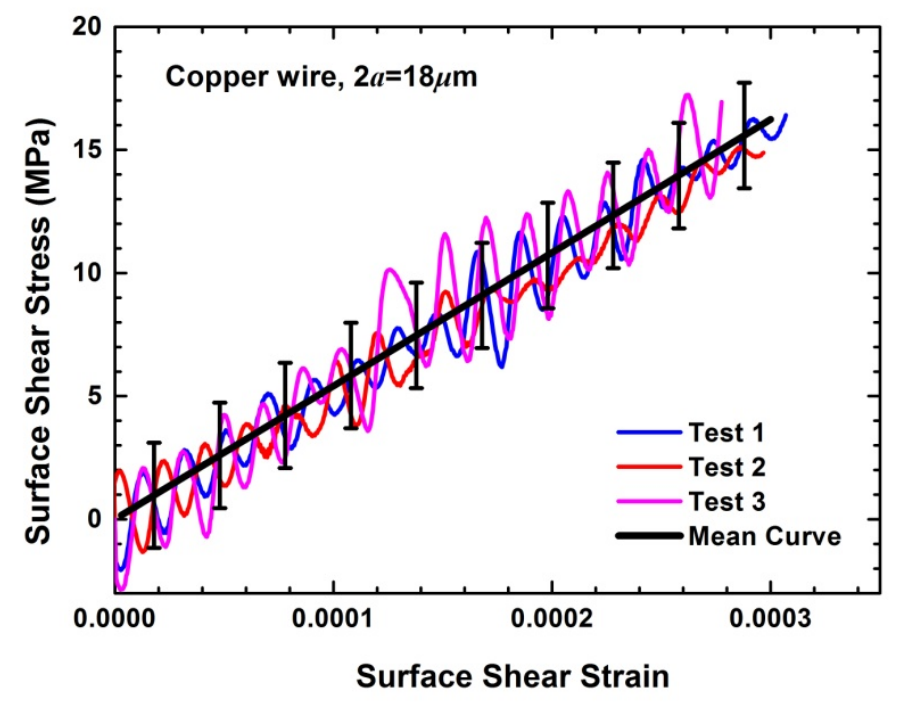

FIG. 5. Torsional stress-strain curves within elastic range for the copper wires of diameter $18 \mu \mathrm{m}$.

Compared with the previous study [1], there are three noteworthy features in the present technique although both are based on the similar measurement principle. Firstly, the misalignment has been a main problem in the previous design since both ends of the torsion 
wire are fixed and the specimen is connected to the torsion wire through a rigid frame. Here, the specimen is straightforwardly connected to the torsion wire via the cross-beam, which improves the coaxiality between the torsion wire and the specimen. Secondly, the cross-beam symmetrical about the torsion wire, paired with a laser displacement sensor, is used as the angle detector for measuring the instantaneous rotation of the torsion wire as the specimen is twisted. Thirdly, the method for preparing the specimen has been improved. By doing these, the error due to the off-axis rotation is greatly reduced. Therefore, the modified design of torsion tester is allowed to measure the torque as low as $10^{-9} \mathrm{~N} \cdot \mathrm{m}$ with a resolution of up to $10^{-10} \mathrm{~N} \cdot \mathrm{m}$, which enables to characterize single fibers with diameter less than $10 \mu \mathrm{m}$. For example, the torsional properties of individual carbon fibers of diameter $7.02 \pm 0.18 \mu \mathrm{m}$ are studied (see Section 4).

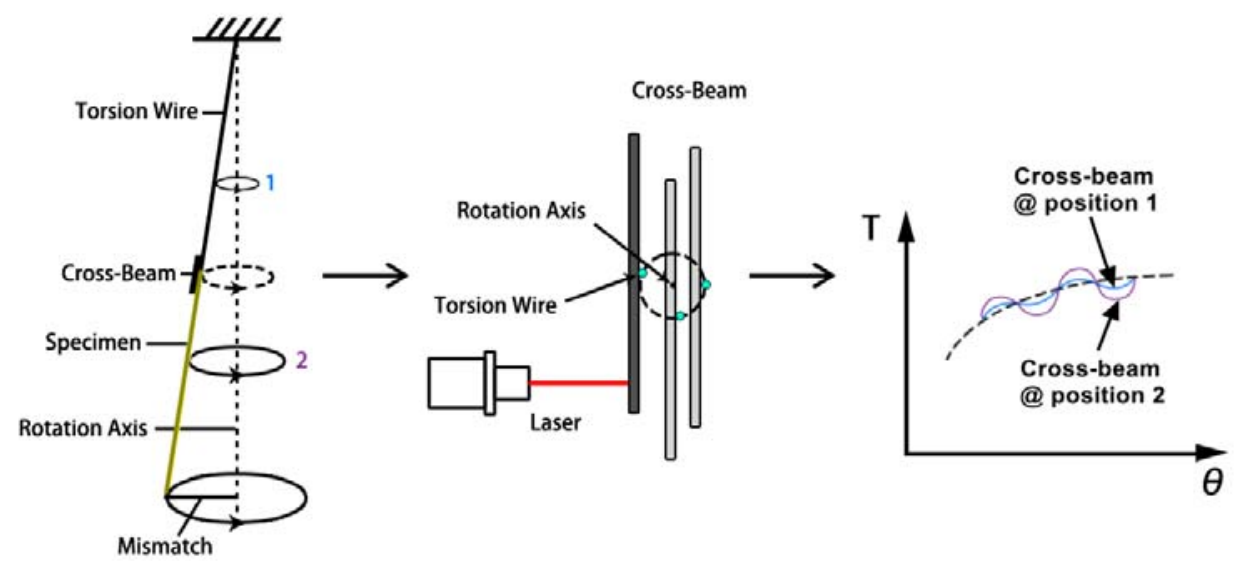

FIG. 6. Influence of the vertical misalignment between a specimen and the twisting head during the measurement. (a) The misalignment at different positions; (b) different positions of the cross-beam during one rotation on its circuit lead to an alternating torque measured by the optical detection system; (c) typical fluctuations in a torque-twist curve.

\section{Results and discussion}

All the torsion measurements were performed in twist-controlled mode and carried out at room temperature. In a twist-controlled mode, the test is conducted in such a way that the twisting rate given by the stepper motor during the test is maintained constant and the torque required is measured. The gauge length of the tested wires was varied between 60 
and $100 \mathrm{~mm}$. The rotating speed of the turntable used here was $6.28 \mathrm{rad} / \mathrm{min}$. Due to the difference of diameter and gauge length of the specimens, the surface shear strain rate of each specimen was not the same, but all were below $3 \times 10^{-5} / \mathrm{s}$.

The shear stress-strain curves for the $50 \mu \mathrm{m}$-diameter copper wires and silver wires are shown in Fig. 7. The curves are scattered but still exhibit reasonably repeatability. We used the mean curve as a representative response. The error bars marked on the mean curve are not results from calculating deviations, which are only used to indicate the upper and lower boundary of the curve scattering. Note that the large perturbations on the curves are caused by the air current. Both the copper wires and the silver wires exhibit linear elastic followed by non-linear yielding behavior at small strains. The elastic shear moduli for these two types of wires are calculated to be $36.84 \pm 2.42 \mathrm{GPa}$ and $25.48 \pm 1.02 \mathrm{GPa}$, respectively. The shear yield strengths for these two types of wires are around $40 \mathrm{MPa}$ and $60 \mathrm{Mpa}$, respectively. These values are in consistent with those in references [26, 27, 29].
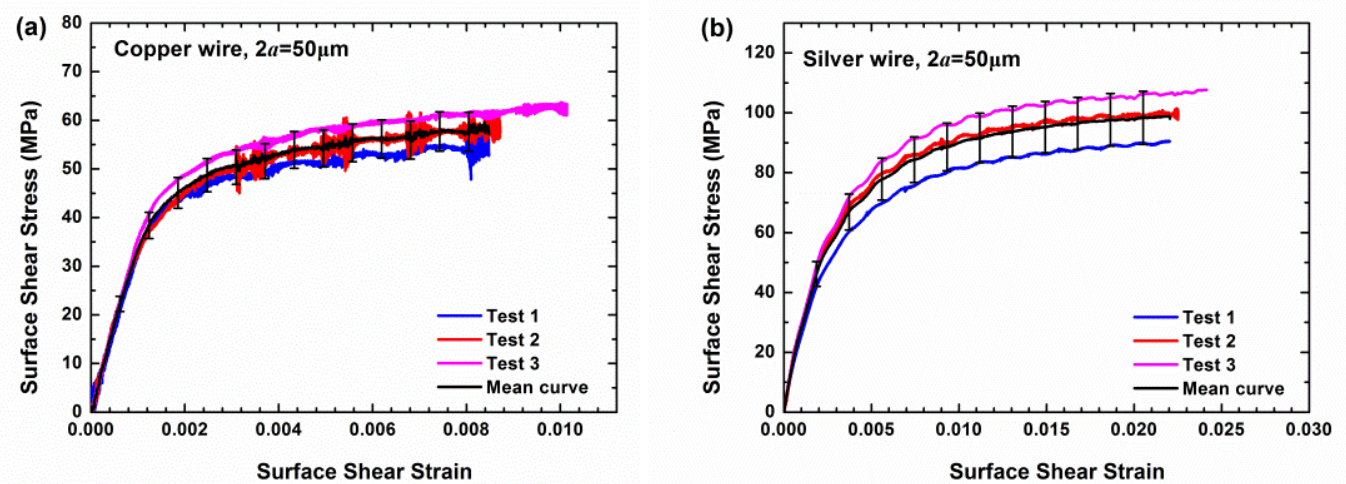

FIG. 7. Torsional response of copper wires and silver wires. (a) Copper wires of diameter 50um; (b) Silver wires of diameter $50 \mu \mathrm{m}$.

To study the capability as well as the resolution of the tester, the torsion tests were also conducted on $7 \mu \mathrm{m}$-diameter carbon fibers by following the same procedure, as shown in Fig. 8. Seven measurements under a same testing condition were repeated. One can see that the response of carbon fiber is fully elastic even for a large shear strain at surface as the carbon fiber is brittle in nature, which has been confirmed by the torsion experiments performed by Sawada and Shindo [30]. The shear modulus of the T300 carbon fibers is measured to be $19.88 \pm 2.42 \mathrm{GPa}$, which is consistent with the published values $[26,27,30,31]$. We have also measured the Young's modulus for the same carbon fibers by a specially designed fiber 
tensile module [32], which gives $E_{\text {carbon }}=235.4 \pm 12.4 \mathrm{GPa}$. Therefore, the resulting Poisson's ratio would be $v=E / 2 G-1=4.9$, indicating that the carbon fiber is anisotropic since the isotropic materials must have a Poisson's ratio of $-1<v<0.5$. This conclusion is in agreement with that obtained by Tsai and Daniel[33] and Behlow et al. [6].

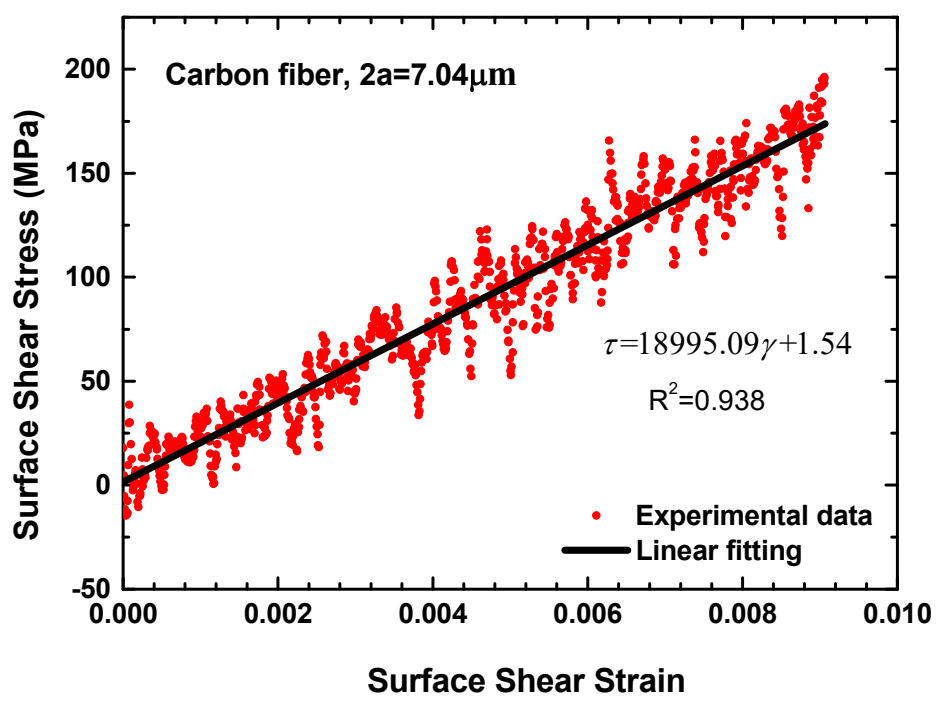

FIG. 8. Typical torsion data on a single T300 carbon fiber (Diameter: $7.02 \pm 0.18 \mu \mathrm{m}$, Length: $66.50 \mathrm{~mm})$. The torsion constant of torsion wire used here is $0.043 \mu \mathrm{N} \bullet \mathrm{cm} / \mathrm{deg}$. The shear modulus of carbon fiber determined by the linear fitting is $18.995 \mathrm{GPa}$.

Table 1. Shear moduli for copper, silver wires and carbon fibers.

\begin{tabular}{lccccc}
\hline Specimen & Diameter $(\mu \mathrm{m})$ & $\begin{array}{c}\text { Diameter of torsion } \\
\text { wire }(\mu \mathrm{m})\end{array}$ & $\begin{array}{c}K \text { of torsion wire } \\
\text { (nN.m/deg) }\end{array}$ & $\begin{array}{c}\text { Measured } G \\
(\mathrm{GPa})\end{array}$ & $\begin{array}{l}G \text { in Refs. } \\
\text { (GPa) }\end{array}$ \\
\hline Copper wires & $18.19 \pm 0.14$ & $30.53 \pm 0.10$ & $6.3-7.1$ & $54.11 \pm 2.14$ & $40-49[6,26,27]$ \\
\hline Copper wires & $49.89 \pm 0.27$ & $40.58 \pm 0.20$ & $32.8-58.8$ & $36.84 \pm 2.42$ & $40-49[6,26,27]$ \\
\hline Carbon fibers & $7.02 \pm 0.18$ & $15.20 \pm 0.10$ & $0.43-0.78$ & $19.88 \pm 2.42$ & $14-22[26,27,30,31]$ \\
\hline
\end{tabular}

*Note that the different values of $K$ for torsion wires with a same diameter are due to the different length of torsion wire we used. 


\section{Conclusions}

A torsion-balance tester has been developed for the measurement of torsional properties of single micron-diameter fibers/fibers. The design of the tester is allowed to perform torque-twist measurement on single fibers/wires under a condition of constant tension. As examples, the torsion tester is applied to investigate the torsional properties of thin metallic wires and carbon fibers of diameter from several microns to tens microns. The experimental results demonstrate that the technique can be used to measure the toque down to $\mathrm{nN} \cdot \mathrm{m}$ regime with a precision of about $8 \%$. Unavoidably, the misalignment between the torsion wire and the twisting head would give some noise in the measured torque-twist curves, which is analyzed in detail. Many torsional parameters, such as the shear modulus and shear yield strength, can be obtained with a reasonable accuracy. For further progress, the proposed technique is expected to be extended to characterize other torsional characteristics, including creep, hysteresis, fatigue, and torque relaxation, etc.

\section{Acknowledgements}

This work was supported by the National Natural Science Foundation of China (No. 11072084 and No. 11272131), the Natural Science Foundation of Hubei Province (No. 2015CFB394) and the Fundamental Research Funds for the Central Universities (HUST, No. 2015QN138). This project has received funding from the European Union's Horizon 2020 research and innovation programme under the Marie Sklodowska-Curie grant agreement No 704292.

\section{References}

[1] Liu D, He Y, Dunstan DJ, Zhang B, Gan Z, Hu P, et al. Toward a further understanding of size effects in the torsion of thin metal wires: An experimental and theoretical assessment. Int J Plasticity 2013;41:30-52.

[2] Dunstan DJ, Galle JU, Ehrler B, Schmitt NJ, Zhu TT, Hou XD, et al. Micromechanical testing with microstrain resolution. Rev Sci Instrum 2011;82:093906.

[3] Fleck NA, Muller GM, Ashby MF, Hutchinson JW. Strain gradient plasticity: Theory and experiment. Acta Met Mater 1994;42:475-87. 
[4] Hutchinson JW. Plasticity at the micron scale. Int J Solids Struct 2000;37:225-38.

[5] Dhingra RC, Postle R. The Measurement of Torque in Continuous-Filament Yarns, Part 1: Experimental Techniques. J Text I 1974;65:126-32.

[6] Behlow H, Saini D, Oliveira L, Durham L, Simpson J, Serkiz SM, et al. Direct measurement of shear properties of microfibers. Rev Sci Instrum 2014;85:095118.

[7] Tan EPS, Lim CT. Novel approach to tensile testing of micro- and nanoscale fibers. Rev Sci Instrum 2004;75:2581.

[8] Han JH, Saif MTA. In situ microtensile stage for electromechanical characterization of nanoscale freestanding films. Rev Sci Instrum 2006;77:045102--8.

[9] Gillies GT, Ritter RC. Torsion balances, torsion pendulums, and related devices. Rev Sci Instrum 1993;64:283-309.

[10] Coulomb CA. Memoires de I'Academie Royale des Sciences. Paris: Academie royale des sciences; 1784.

[11] Cavendish H. Experiments to Determine the Density of the Earth. Philosophical Transactions of the Royal Society of London 1798:469-526.

[12] Peirce FT. The Plasticity of Cotton and Other Materials. J Text I T 1923;14:T390-T413.

[13] Huan Y, Dai Y, Shao Y, Peng G, Feng Y, Zhang T. A novel torsion testing technique for micro-scale specimens based on electromagnetism. Rev Sci Instrum 2014;85:095106.

[14] Mitchell TW, Feughelman M. The Torsional Properties of Single Wool Fibers Part I: Torque-Twist Relationships and Torsional Relaxation in Wet and Dry Fibers. Text Res J 1960;30:662-7.

[15] Postle R, Burton P, Chaikin M. The torque in twisted singles yarns. J Text I T 1964;55:T448-T61.

[16] Armstrong LD, Mitchell TW. The application of a light-sensitive potentiometer in the measurement of the mechanical properties of single fibres. Journal of Physics E: Scientific Instruments 1969;2:375.

[17] Postle R, Burton P, Chaikin M. The Torque in Twisted Singles Yarns. J Text I T 1964;55:T448-T61.

[18] Skelton J. The measurement of the torsional elastic recovery of filaments. J Text I T 1965;56:T443-T53.

[19] Kawabata S. Measurements of anisotropic mechanical property and thermal conductivity of single fiber for several high performance fibers. The 4 th Japan-U S Conference on Composite Materials Washington1989. p. 253-62.

[20] Mccord MG, Ellison MS. An automated torsion balance for investigation of microstructure of single filaments. I. Polypropylene. J Appl Polym Sci 1996;61:293-306.

[21] Song B, Lu W-Y. An Improved Experimental Technique to Characterize Micro-Diameter Copper Wires in Torsion. Exp Mech 2015;55:999-1004.

[22] Liu D, He Y, Tang X, Ding H, Hu P. Size effects in the torsion of microscale copper wires: Experiment and analysis. Scripta Mater 2012;66:406-9.

[23] Walter M, Kraft O. A new method to measure torsion moments on small-scaled specimens. Rev Sci Instrum 2011;82:035109.

[24] Dunstan DJ, Ehrler B, Bossis R, Joly S, P'NG KMY, Bushby AJ. Elastic limit and strain hardening of thin wires in torsion. Phys Rev Lett 2009;103:155501.

[25] Bushby AJ, Dunstan DJ. Size effects in yield and plasticity under uniaxial and non-uniform loading: Experiment and theory. Phil Mag 2011;91:1037-49.

[26] Liu D, He Y, Hu P, Gan Z, Ding H. A modified torsion pendulum for measuring the shear modulus of single micro-sized filament. Acta Mech Solida Sin 2014;27:221-32. 
[27] Yu L, Liu D, Peng K, He Y. An improved torsion pendulum based on image processing for single fibers. Meas Sci Technol 2016;27:075601.

[28] Tavanai H, Denton MJ, Tomka JG. Direct objective measurement of yarn-torque level. J Text I 1996;87:50-8.

[29] Lu WY, Song B. Quasi-Static Torsion Characterization of Micro-diameter Copper Wires. Exp Mech 2010;51:729-37.

[30] Sawada Y, Shindo A. Torsional properties of carbon fibers. Carbon 1992;30:619-29.

[31] Adams RD. The dynamic longitudinal shear modulus and damping of carbon fibres. J Phys D: Appl Phys 1975;8:738-48.

[32] Liu D, He Y, Hu P, Ding H, Tang X, Li Z. On the mechanical properties in micro-tensile testing of single fiber. J Exp Mech 2012;27:61-9.

[33] Tsai CL, Daniel IM. Determination of shear modulus of single fibers. Exp Mech 1999;39:284-6. 